\title{
AN INFINITESIMAL PROOF OF THE IMPLICIT FUNCTION THEOREM
}

\author{
by NIGEL J. CUTLAND and FENG HANQIAO
}

(Received 11 November, 1991)

We give a short and constructive proof of the general (multi-dimensional) Implicit Function Theorem (IFT), using infinitesimal (i.e. nonstandard) methods to implement our basic intuition about the result. Here is the statement of the IFT, quoted from [4];

Theorem. Let $A \subset \mathbb{R}^{n} \times \mathbb{R}^{m}$ be an open set and let $F: A \rightarrow \mathbb{R}$ be a function of class $C^{p}$ $(p \geq 1)$. Suppose that $\left(x_{0}, y_{0}\right) \in A$ with $F\left(x_{0}, y_{0}\right)=0 \quad\left(x_{0} \in \mathbb{R}^{n}, y_{0} \in \mathbb{R}^{m}\right)$ and that the Jacobian determinant $J=\frac{\partial\left(F_{1}, \ldots, F_{m}\right)}{\partial\left(y_{1}, \ldots, y_{m}\right)}$ is not zero at $\left(x_{0}, y_{0}\right)$. Then there is an open neighbourhood $U$ of $x_{0}$ and a unique function $f: U \rightarrow \mathbb{R}^{m}$ with

$$
F(x, f(x))=0
$$

for all $x \in U$. Moreover, $f$ is of class $C^{p}$.

First let us give an intuitive informal description of $f$; we need some notation. Points $x, y \in \mathbb{R}^{n}, \mathbb{R}^{m}$ will be regarded as column vectors; we write $\partial F / \partial y$ for the $m \times n$ Jacobian matrix $\partial F / \partial y=\left(\partial F_{i} / \partial y_{j}\right)$, where we have $F=\left(F_{1}, \ldots, F_{m}\right)^{\prime}$ and $F_{i}=F_{i}(x, y)$. Then $J=|\partial F / \partial y|$. Similarly $\partial F / \partial x=\left(\partial F_{i} / \partial x_{j}\right)$, an $m \times n$ matrix.

Intuitively, a recipe for $f$ is given as follows. Writing $d x=\left(d x_{1}, \ldots, d x_{n}\right)^{\prime}$ etc., we have, informally

$$
0=d F(x, f(x))=\frac{\partial F}{\partial x} d x+\frac{\partial F}{\partial y} d f
$$

If $\partial F / \partial y$ is invertible (which it is in a neighbourhood of $\left(x_{0}, y_{0}\right)$ ) then

$$
d f(x)=f(x+d x)-f(x)=-\frac{\partial F^{-1}}{\partial y} \frac{\partial F}{\partial x} d x .
$$

Using infinitesimal techniques we can implement this recipe for $f$, by discretizing the space $\mathbb{R}^{n}$ and using (1) as a recursive definition for $f$. We assume the basics of nonstandard analysis, which may be found in [1] or [3].

Pick a positive infinitesimal $\Delta \neq 0$ and let $T=\left\{k \Delta: k \in{ }^{*} \mathbb{Z}\right\}$. We will consider $\tau=\left(t_{1}, \ldots, t_{n}\right)$ taking values in the lattice $T^{n} \subseteq{ }^{*} \mathbb{R}^{n}$.

We shall need the following elementary lemma [2].

Lemma. Let $\psi: T \rightarrow{ }^{*} \mathbb{R}$ be internal, and let $D \psi$ be the difference function:

$$
D \psi(t)=\frac{\psi(t+\Delta)-\psi(t)}{\Delta} .
$$

If $\psi(0)$ is finite and $D \psi$ is $S$-continuous for $|t| \leq c$ then there is a unique standard function $g:[-c, c] \rightarrow \mathbb{R}$ given by

$$
g\left({ }^{\circ} t\right)={ }^{\circ} \psi(t)
$$

Glasgow Math. J. 35 (1993) 163-166. 
Moreover, $g$ is $C^{1}$, and $D g\left({ }^{\circ} t\right)={ }^{\circ} D \psi(t)$. (Recall that $\psi$ is S-continuous if $\psi(t) \approx \psi\left(t^{\prime}\right)$ whenever $t \approx t^{\prime}$.)

Proof of the IFT Without loss of generality we may assume that $x_{0}=0$ and $y_{0}=0$. Define an internal function $\varphi: T^{n} \rightarrow{ }^{*} \mathbb{R}^{m}$ recursively as follows.

(i) $\varphi(0)=0$

(ii) for each $0<k \leq n$ and $\sigma \in T^{k-i}$, if $\varphi(\sigma, 0, \ldots, 0)=\varphi\left(\sigma_{1}, \ldots, \sigma_{k-1}, 0, \ldots, 0\right)$ has been defined, then define $\varphi(\sigma, t, \ldots, 0)$ for $t \in T$ by:

$$
\begin{aligned}
& \varphi(\sigma, t+\Delta, \ldots, 0)=\varphi(\sigma, t, \ldots, 0)-\frac{\partial F^{-1}}{\partial y} \frac{\partial F}{\partial x_{k}} \Delta \text { if } t \geq 0 \\
& \varphi(\sigma, t-\Delta, \ldots, 0)=\varphi(\sigma, t, \ldots, 0)+\frac{\partial F^{-1}}{\partial y} \frac{\partial F}{\partial x_{k}} \Delta \text { if } t \leq 0 .
\end{aligned}
$$

Note that by Cramer's rule, this explicit recipe is given by

$$
\varphi_{i}(\sigma, t \pm \Delta, \ldots, 0)=\varphi_{i}(\sigma, t, \ldots, 0) \mp \Delta J^{-1} \frac{\partial\left(F_{1}, \ldots, F_{m}\right)}{\partial\left(y_{1}, \ldots, y_{i-1}, x_{k}, y_{i+1}, \ldots, y_{m}\right)} .
$$

The matrices $\partial F / \partial y$ and $\partial F / \partial x$ are evaluated at $x=(\sigma, t, \ldots, 0)$ and $y=\varphi(x)$. The hypotheses on $\partial F / \partial x$ and $\partial F / \partial y$ ensure that on some rectangle $-a \leq x_{i}, y_{i} \leq a$ (where $a$ is positive standard) there is a standard $M>0$ with $\left|\left(\frac{\partial F^{-1}}{\partial y} \frac{\partial F}{\partial x}\right)_{j, k}\right| \leq M$ for all $j, k$. It is easy to check that this ensures that for $\tau=\left(t_{1}, \ldots, t_{n}\right)$ with each $\left|t_{i}\right| \leq \frac{a}{M n}$ the above definition gives $\left|\varphi_{j}(\tau)\right| \leq a$. (This is done by induction, as in the definition of $\varphi$ : in fact we show that if each $\left|t_{i}\right| \leq \frac{a}{M n}$ then for each $k \leq n$ we have

$$
\left|\varphi_{j}\left(t_{1}, \ldots, t_{k}, 0, \ldots, 0\right)\right| \leq \frac{k a}{n}
$$

If (2) holds for $k$, the definition of $\varphi$ ensures that if $|t| \leq \frac{a}{M n}$ then

$$
\left|\varphi_{j}\left(t_{1}, \ldots, t_{k}, t, \ldots, 0\right)-\varphi_{j}\left(t_{1}, \ldots, t_{k}, 0, \ldots, 0\right)\right| \leq M|t| \leq \frac{a}{n}
$$

which is sufficient to establish (2) for $k+1$.)

Let $b=\frac{a}{M n}$ and for $\tau=\left(t_{1}, \ldots, t_{n}\right) \in T^{n}$ write $|\tau| \leq b$ to mean $\left|t_{i}\right| \leq b$ for all $i$. It is clear from the definition of $\varphi$ that

$$
\left|\varphi\left(t_{1}, \ldots, t_{k}, t, 0, \ldots, 0\right)-\varphi_{j}\left(t_{1}, \ldots, t_{k}, t^{\prime}, 0, \ldots, 0\right)\right| \leq M\left|t-t^{\prime}\right|
$$

for $|\tau| \leq b$ and $|t|,\left|t^{\prime}\right| \leq b$. In particular $\varphi\left(t_{1}, \ldots, t_{n}\right)$ is $S$-continuous in $t_{n}$ for $\left|t_{i}\right| \leqslant b$. We will show later that it is $S$-continuous in all its arguments.

We now show that

$$
F(\tau, \varphi(\tau)) \approx 0 \text { for }|\tau| \leq b, \quad \tau \in T^{n}
$$


This is again done by induction as in the definition of $\varphi$. Let $\tau=(\sigma, t, \ldots, 0)$ and $\tau^{\prime}=(\sigma, t+\Delta, \ldots, 0)$. Then by the mean value theorem

$$
F_{j}\left(\tau^{\prime}, \varphi\left(\tau^{\prime}\right)\right)-F_{j}(\tau, \varphi(\tau))=\frac{\partial F_{j}}{\partial x_{k}}(\bar{\tau}, \bar{\eta}) \Delta+\frac{\partial F_{j}}{\partial y}(\bar{\tau}, \bar{\eta})\left(\varphi\left(\tau^{\prime}\right)-\varphi(\tau)\right)
$$

for some $\bar{\tau}$ between $\tau$ and $\tau^{\prime}$, and $\bar{\eta}$ between $\varphi(\tau)$ and $\varphi\left(\tau^{\prime}\right)$. Now use the definition of $\varphi$ to see that

$$
F_{j}\left(\tau^{\prime}, \varphi\left(\tau^{\prime}\right)\right)-F_{j}(\tau, \varphi(\tau))=\left[\frac{\partial F_{j}}{\partial x_{k}}(\bar{\tau}, \bar{\eta})-\frac{\partial F_{j}}{\partial y}(\bar{\tau}, \bar{\eta})\left(\frac{\partial F^{-1}}{\partial y} \frac{\partial F}{\partial x_{k}}\right)(\tau, \varphi(\tau))\right] \Delta=\Delta \epsilon
$$

where $\epsilon \approx 0$ by the continuity of all derivatives of $F$, and the fact that $\tau \approx \tau^{\prime}$ and $\varphi(\tau) \approx \varphi\left(\tau^{\prime}\right)$ by (3). Now $\epsilon$ depends on $\tau$, but we may take $\epsilon_{0}=$ maximum of all $\epsilon$ as $\tau$ varies in $|\tau| \leqslant b$, and then it is easy to see that $F_{j}(\tau, \varphi(\tau)) \approx F_{j}(0, \varphi(0))=0$ for all such $\tau$.

We now see that $\varphi$ is essentially unique with the property (4). We show that

$$
F(\tau, y) \approx F\left(\tau, y^{\prime}\right) \Rightarrow y \approx y^{\prime}
$$

for $|\tau|,|y|,\left|y^{\prime}\right| \leq b$. By the mean value theorem

$$
0 \approx F_{j}\left(\tau, y^{\prime}\right)-F_{j}(\tau, y)=\frac{\partial F_{j}}{d y}\left(\tau, y^{j}\right)\left(y^{\prime}-y\right)
$$

for some $y^{j} \in \mathbb{R}^{m}$ between $y$ and $y^{\prime}$. Now the assumption $J(0,0) \neq 0$ and continuity of derivatives means that for small enough $a$, and $|\tau|,|y|,\left|y^{\prime}\right| \leq a$ the matrix $\left(\frac{\partial F_{j}}{\partial y}\left(\tau, y_{j}\right)\right)$ is non-singular, and so $y^{\prime} \approx y$.

To show that $\varphi$ is $S$-continuous in all its arguments, fix $k<n$ and consider another function $\bar{\varphi}$ defined like $\varphi$ but with indices $1, \ldots, n$ permuted so that $k$ is the last. Then the above all applies to $\bar{\varphi}$ : in particular, from (4)

$$
F(\tau, \bar{\varphi}(\tau)) \approx 0 \text { for }|\tau| \leq b, \quad \tau \in T^{n}
$$

and so from (5)

$$
\varphi(\tau) \approx \bar{\varphi}(\tau) \quad \text { all } \quad \tau=\left(t_{1}, \ldots, t_{k}\right), \quad|\tau| \leq b .
$$

Moreover, $\bar{\varphi}\left(t_{1}, \ldots, t_{n}\right)$ is $S$-continuous in $t_{k}$, and hence $\varphi$ is $S$-continuous in $t_{k}$. Thus $\varphi$ is $S$-continuous on $|\tau| \leq b$ and we can define a standard continuous function $f$ by

$$
f\left({ }^{\circ} \tau\right)={ }^{\circ} \varphi(\tau) \quad|\tau| \leq b, \quad \tau \in T^{n} .
$$

From (4) and the continuity of $F$, we have

$$
F(x, f(x))=0 \text { for }|x| \leq b .
$$

The uniqueness of $f$ for $|x| \leq b$ is given by the argument used to give (5).

To see that $f$ is continuously differentiable, the definition of $\varphi$ together with the lemma shows that

$$
\frac{\partial f}{\partial x_{k}}\left(x_{1}, \ldots, x_{k}, 0, \ldots, 0\right)=-\frac{\partial f^{-1}}{\partial y} \frac{\partial F}{\partial x_{k}}(x, f(x))
$$


(where $x=\left(x_{1}, \ldots, x_{k}, 0, \ldots, 0\right)$ ). A simple symmetry argument shows that this is valid for all $x$ with $|x| \leq b$; i.e.

$$
\frac{\partial f}{\partial x}=-\frac{\partial F^{-1}}{\partial y} \frac{\partial F}{\partial x}(x, f(x))
$$

for all $x$ with $|x| \leq b$. If $F$ is $C^{p}$, repeated differentiation shows that $f$ is also $C^{p}$.

\section{REFERENCES}

1. N. J. Cutland, (Editor), Nonstandard analysis and its applications (Cambridge University Press, 1988).

2. Feng Hanqiao, D. F., St. Mary and F. Wattenberg, Applications of nonstandard analysis to partial differential equations-the diffusion equation, Mathematical Modelling, 7 (1986) 507-523.

3. A. E. Hurd and P. A. Loeb, An introduction to nonstandard real analysis (Academic Press, 1985).

4. J. E. Marsden, Elementary classical analysis (W. H. Freeman, 1974).

Department of Pure Mathematics

UNIVERSITY OF HULL

HuLl HU6 7RX

ENGLAND
Department of Computer Science SHAANXI UNIVERSITY

XIAN, 710062

China 